\title{
sciendo
}

\author{
Current Issues in Pharmacy and Medical Sciences
}

Formerly ANNALES UNIVERSITATIS MARIAE CURIE-SKLODOWSKA, SECTIO DDD, PHARMACIA

\section{Walnut Kernel administration to mothers during pregnancy and lactation improve learning of their pups. Changes in number of neurons and gene expression of NMDA receptor and $\mathrm{BDNF}$ in hippocampus in $\mathbf{8 0}$ days rat pups}

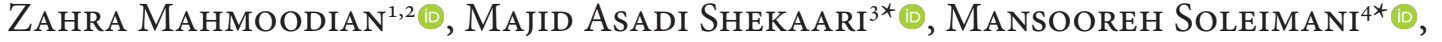

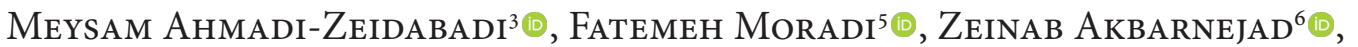 \\ Khadijeh Esmaeilpour ${ }^{3}$, ${ }^{\text {Taj Pri Kalantaripour }}{ }^{70}$
}

${ }^{1}$ International Campus, Iran University of Medical Sciences, Tehran, Iran

${ }^{2}$ Department of Cell Biology Research Center, Iran University of Medical Sciences, Tehran, Iran

${ }^{3}$ Neuroscience Research Center, Neuropharmacology Institute, Kerman University of Medical Sciences, Kerman, Iran

${ }^{4}$ Cellular and Molecular Research Center, Iran University of Medical Sciences, Tehran, Iran

${ }^{5}$ Department of Anatomical Science, Iran University of Medical Sciences, Tehran, Iran

${ }^{6}$ ENT and Head \& Neck Research Center and Department, Hazrat Rasoul Hospital, The Five Senses Institute, Iran University of Medical Sciences, Tehran, Iran

7 Department of Basic Sciences, School of Medicine, Branch of Kerman, Islamic Azad University, Kerman, Iran

\section{ARTICLE INFO}

Received 25 August 2019

Accepted 04 June 2020

\section{Keywords:}

rat,

memory,

Walnut,

learning,

BDNF,

NMDA-R,

offspring.

\begin{abstract}
Walnut (Juglans regia) from the Juglandaceae family contains high levels of omega 3 fatty acid, vitamin E and melatonin, hence its consumption is beneficial to would be mothers and their offspring. The current study was designed to determine the possible mechanism of walnut consumption by mothers during pregnancy and lactation and the positive effects on learning and memory processes in their offspring. Wistar adult female rats were placed into three groups: control (fed with pellet, $20 \mathrm{~g}$ daily during pregnancy and lactation), CASE 1 [fed with Walnut Kernel (WK) 6\% of food intake during pregnancy and lactation] and CASE 2 (fed with WK, $9 \%$ of food intake during gestation and lactation). In order to evaluate offspring learning and memory, the Morris Water Maze (MWM) test was performed for their adult offspring at 80 days of age. Histological and molecular studies were utilized in order to discover the protective mechanism and efficacy of WK consumption. The results revealed that learning was significantly improved in the females of CASE 2, in comparison to controls, while there was no difference in memory among the different groups. In addition, the number of neurons significantly increased in the CASE 2 group compared to the control group. However, the molecular study demonstrated that there was no significant difference among the study groups. The results herein show that feeding mothers with WK may improve the learning competence of their pups and increase the number of neurons in both sexes.
\end{abstract}

\section{INTRODUCTION}

Generally, the greatest effects on mothers and children occurs in the "first 1000 days" from the beginning of pregnancy to the second year of birth [1]. Different studies have shown that brain synapse growth rapidly occurs in the first years of life. Nutrition from prenatal period to the end of the second year of birth has, therefore, a critical role in brain

\footnotetext{
* Corresponding author

e-mail:majidasadi@kmu.ac.ir, soleimanim@aut.ac.ir
}

formation and development [2]. Some studies have shown that the quality and quantity of fatty acid consumption during pregnancy considerably affect the pre and post-natal period. Docosahexaenoic acid (DHA) is the most important factor for fetal growth and development. It is responsible for membrane fixation, neuroplasticity, signal transmitting and neurotransmitter density regulation. Accumulation of DHA in the brain occurs at high speed in the third trimester up to five years of age. Moreover, the precursor of polyunsaturated 
fatty acid (PUFA) long chains such as Arachidonic Acid (AA) and DHA is selectively transferred from the placenta to the fetus during pregnancy $[3,4]$.

Nuts contain several neuroprotective agents (among others, vitamin E, folate, polyphenol and Omega-3 PUFA) that are involved in brain activity and affect cognitive processes [5]. Juglans regia is not merely an agriculture product, but its fruit, leaves, seeds and flowers are used for diverse medicine purposes worldwide [6]. It has been reported that walnut consumption in old rats improves memory and cognitive actions $[6,7]$. In addition, walnut has numerous anti-epileptic, neuroprotective and anti-oxidant effects [8]. Asadi et al. (2013) showed, for example, that walnut consumption during pregnancy and lactation affects the learning and memory of offspring [9]. This is because the lower part of the limbic system that is located in the middle of the temporal lobe of the human brain (the hippocampus) [10] consists of pyramidal neurons in the CA1 area with a euchromatic nucleus that is critical for learning and memory processes [11], and hippocampus growth commences from the prenatal period [12].

Brain-derived neurotrophic factor (BDNF) is a gene of the neurotrophins family with a critical role in brain growth and development, learning and spatial memory [13]. N-Methyl-D-Aspartate Receptor (NMDA-R) in the brain and spinal cord acts as a mediator for most of neurotransmitters. Glutamate has different receptors, including ionotropic glutamate receptors, that are divided into three large families, $\alpha$-amino-3-hydroxy-5-methyl-4-isoxazolepropionic acid (AMPA), kainite and NMDA. Sub-units of NMDA are involved in the synapse of the pyramidal cells of the CA1 region of the hippocampus to form and integrate synapse [14]. In line with our previous study, we decided to investigate the possible molecular mechanism of WK consumption by mothers (during pregnancy and lactation) and its positive effects on learning and memory processes in their offspring.

\section{MATERIALS AND METHODS}

\section{Animals}

In this study, nine adult female Wistar rats weighing 200-250 g were used. The Ethics Committee at Kerman University of Medical Sciences approved the procedures for this study (EC/KNRC/93-66). Animals were housed in standard conditions, including the temperature of $22 \pm 2^{\circ} \mathrm{C}$ and $25 \%$ humidity, $12 \mathrm{~h}$ light-dark cycle, free access to food and water ad libitum. Pairs of females were then placed in the same cage with a single male rat in the late afternoon. Vaginal smears were examined the following morning. The day on which any sperm was found was designated as the gestation day of 0 (GD 0 ). After this, pregnant rats were randomly divided into three groups ( $\mathrm{n}=3$ in each group): control (fed with pellet, $20 \mathrm{~g}$ daily), gestation and lactation CASE 1 (fed with WK, 6\% of food intake during pregnancy and lactation), and gestation and lactation CASE 2 (fed with WK, 9\% of food intake during gestation and lactation). The WK was freshly prepared from high-quality walnuts and removed from the shell at the time of feeding. The duration of pregnancy and lactation was three weeks each. The weight of the rats was recorded daily. After the feeding period, behavioral tests were conducted for pups (at least $n=8$ ), using the MWM test at the age of 80 days [9].

\section{Plant Material}

The WK was collected from Rabor area, Kerman province, Iran in September 2010. A voucher specimen was deposited at the herbarium of the Faculty of Pharmacy, Kerman University of Medical University (No. 1401-1).

\section{Learning and memory evaluation using Morris water maze test}

Learning and memory was evaluated at the age of 80 days using the MWM test through which rats learned to escape a water basin onto a hidden platform. The maze consisted of a round steel tub (1.36 $\mathrm{m}$ in diameter and $60 \mathrm{~cm}$ high) filled with water at the temperature of $22 \pm 1{ }^{\circ} \mathrm{C}$. Animals were challenged to find a hidden platform $(10 \mathrm{~cm}$ in diameter and $25 \mathrm{~cm}$ high) located $1.5 \mathrm{~cm}$ below water level $(26.5 \mathrm{~cm})$ and in the same place during the entire trial. The platform was always positioned $30 \mathrm{~cm}$ beyond the rim of the pool in the center of one quadrant with respect to the distal visual cues. Swimming patterns were analyzed, and the following factors were computed: time spent in traveling in the target quadrant, the distance traveled in the target quadrant (centimeters), and the swimming speed $(\mathrm{cm} / \mathrm{s})$. The experiment was performed over four consecutive days to test the animals' learning. In each trial, the rats were placed in the water facing the rim and began the test from one of four points in the pool, with the platform kept in the same place and with a $1 \mathrm{~min}$ interval between each trial. The rats were trained in the maze during 16 trials, four sessions on consecutive four days, except for the last day.

The animals had to swim to find the hidden platform in $90 \mathrm{~s}$ (maximum time), where they were allowed to stay for $30 \mathrm{~s}$. If they failed to find the platform, they would be placed on it for $10 \mathrm{~s}$ to associate its location with the spatial cues of the room (pictures, cabinets, and light source). One day after the last learning trial, the animals underwent a probe trial to test their long-term spatial memory. On the fifth day, the rats were released in a non-platform pool to check their spatial memory. For this purpose, the percentage of time spent in the target quadrant was recorded and analyzed. In both stages, the movements of the rats in the water were recorded by a camera positioned above the center of the water maze and data were collected by a computer equipped with water maze software [9].

\section{Hippocampus Extraction}

After performing the evaluation by MWM at the age of 80 days, in a stress-free situation, rat offspring ( $\mathrm{n}=3$ male and $\mathrm{n}=3$ female pups for each mother) were anaesthetized intraperitoneally by chloral hydrate $(400 \mathrm{mg} / \mathrm{kg})$. The animals were then decapitated, the hippocampus was extracted, and both whole hippocampi were rapidly dissected out on ice. The right part of hippocampus was placed in formalin 10\% and the left one was dipped into a nitrogen tank and stored at $-80^{\circ} \mathrm{C}$ until homogenization. 


\section{Tissue Processing and H\&E Staining}

For light microscopy studies, following routine procedures, the brains (right hemisphere) were processed according to standard histological methods. The paraffinized brains were cut into $5 \mu \mathrm{m}$ sections on a rotary microtome and the sections were measured by staining them with H\&E using the standard method [15]. Neuronal counting was performed for each animal as the number of pyramidal neurons in three distinct subfields of hippocampal CA1 in coronal sections.

\section{RT-PCR (Reverse Transcriptase-PCR)}

After the extraction of RNA and creation of cDNA, an optimal annealing temperature was selected. Following PCR reaction, proper amounts were placed on gel and electrophoresis was performed. It was then photographed via gel documentary. Subsequently, total cellular RNAs were isolated by applying the chloroform-isopropanol-ethanol procedure, using $\mathrm{RNX}+$ reagent (Cinaclone, Tehran, Iran). After this, the isolated RNA was solved in $20 \mathrm{ml}$ of RNasefree water. Briefly, to produce a PCR template, single-strand cDNA was synthesized from $6.5 \mu$ of purified total RNA using M-Mulv reverse transcriptase and oligo-dT primer (Fermentas, Vilnius, Lithuania). PCR was performed in a final volume of $50 \mu \mathrm{l}$ containing $3 \mu \mathrm{l}$ of tissue cDNA, $1 \mu \mathrm{l}$ of each BDNF, NMDA-R, $\beta$-actin sense and antisense primers, $2 \mu \mathrm{l}$ of dNTP mixture, $1.5 \mu \mathrm{l}$ of $\mathrm{MgCl}_{2}, 5 \mu \mathrm{l}$ of $10 \times$ PCR buffer, $0.3 \mu \mathrm{l}$ of Taq DNA polymerase, and $36.2 \mu \mathrm{l}$ of distilled water. Three separate PCR reactions were conducted to study gene expression in the samples obtained from each rat. Each PCR reaction was performed using selective forward and reverse primers for BDNF, NMDA-R, and $\beta$-actin genes (as an internal standard). The sequences of the primers of gene expression were:

NMDA-R-Forward5'TTACCTTTGAGTCGCCCCTG', NMDAR-Reverse

5'CTGAGCAACGTCTGAGGGTC3',BDNF-Forward 5'GACGACGACGTCCCTGGCTGA3',BDNF-Reverse 5'ACGACTGGGTAGTTCGGCACTGG3', $\beta$-actin-Forward 5'CCCAGAGCAAGAGAGGCATC3', $\beta$-actin-Reverse 5'CTCAGGAGGAGCAATGATCT3'.

All sequences of primers were synthesized by Metabion International AG (Martinsried, Germany). Taq DNA polymerase (Roche, Germany) was utilized for DNA amplification, and reactions were set up according to the manufacturer's protocol. PCR amplification ( 32 cycles) was performed in the following way: initial denaturation for $5 \mathrm{~min}$ at $95^{\circ} \mathrm{C}$, denaturation for $45 \mathrm{~s}$ at $95^{\circ} \mathrm{C}$, annealing for $45 \mathrm{~s}$ at $55.5^{\circ} \mathrm{C}$ for $\beta$-actin, $56.5^{\circ} \mathrm{C}$ for NMDA-R, $60^{\circ} \mathrm{C}$ for BDNF, extension for $45 \mathrm{~s}$ at $72^{\circ} \mathrm{C}$, and final extension for $5 \mathrm{~min}$ at $72^{\circ} \mathrm{C}$. The PCR products were subsequently analyzed on $1 \%$ agarose LMMP (Roche, Germany) gel and the resulting bands were quantified by densitometry (Image $\mathrm{J}$ laboratory work analyzing software).

\section{Statistical analysis}

Data analysis was performed in the Statistical Package for Social Sciences (SPSS) version 16 as Mean \pm SEM, using one-way ANOVA followed by Tukey's post-hoc test. Here, $\mathrm{p}<0.05$ was considered statistically significant.

\section{RESULTS}

\section{Weight}

There was no significant difference between rat offspring weight across groups.

\section{Effect of maternal feeding with WK on learning parameters in adult pups}

There was a significant difference between females of CASE 2 and control in the learning phase $(p \leq 0.05)$. The distance traveled to find the underlying platform and the time to reach the hidden platform in this test also showed a significant difference in the females of CASE 2 group in comparison to the other groups.

In the overall comparison, females of CASE 2 demonstrated better learning than those in CASE 1 and control groups $(\mathrm{p} \leq 0.05)$. These results indicate that the use of WK in pregnancy and lactation improves the learning of the children in the MWM test. However, animal velocity in finding a hidden platform did not show any significant difference across groups (Table 1).

Table 1. Effects of WK consumption by mothers during pregnancy and lactation on their offspring's learning in the MWM test in three groups: as Control: (fed with $20 \mathrm{~g}$ of pellet), CASE 1: (fed with $\mathrm{WK}, 6 \%$ of food intake during pregnancy and lactation), CASE 2: (fed with WK, $9 \%$ of food intake during pregnancy and lactation)

\begin{tabular}{|l|c|c|c|c|c|c|}
\hline & \multicolumn{2}{|c|}{ Distance $(\mathrm{cm})$} & \multicolumn{2}{c|}{ Time $(\mathrm{sec})$} & \multicolumn{2}{c|}{ Velocity $(\mathrm{cm} / \mathrm{s})$} \\
\hline \multirow{2}{*}{ Mean (std) } & Female & Male & Female & Male & Female & Male \\
\hline \multirow{2}{*}{ Control } & 61.2 & 64.98 & 64.21 & 68.12 & 21.44 & 20.49 \\
& $( \pm 1.7)$ & $( \pm 0.9)$ & $( \pm 1.2)$ & $( \pm 2.0)$ & $( \pm 1.4)$ & $( \pm 1.5)$ \\
\hline \multirow{2}{*}{ Case 1 } & 55.35 & 59.65 & 59.45 & 61.93 & 22.69 & 21.25 \\
& $( \pm 0.9)$ & $( \pm 0.5)$ & $( \pm 2.5)$ & $( \pm 1.7)$ & $( \pm 1.9)$ & $( \pm 1.9)$ \\
\hline Case 2 & 28.54 & 52.35 & 29.96 & 55.87 & 23.17 & 23.51 \\
& $( \pm 1.0)^{*}$ & $( \pm 0.7)$ & $( \pm 0.9)^{*}$ & $( \pm 1.0)$ & $( \pm 1.1)$ & $( \pm 1.0)$ \\
\hline
\end{tabular}

The table represents the mean distance $(\mathrm{cm})$, time (seconds) and velocity $(\mathrm{cm} / \mathrm{s})$ of four consecutive days to find the location of the hidden platform between the control group and the cases. The comparison of the groups was performed by one-way ANOVA followed by Tukey 's test. The data are average \pm SD. Females in CASE 2 that consumed more WK ( $9 \%$ of daily intake) than the other groups took less distance and less time to find the hidden platform, which is associated with a significant difference $(\mathrm{p} \leq 0.05)$

\section{Effect of maternal feeding with WK on memory parameters in adult pups}

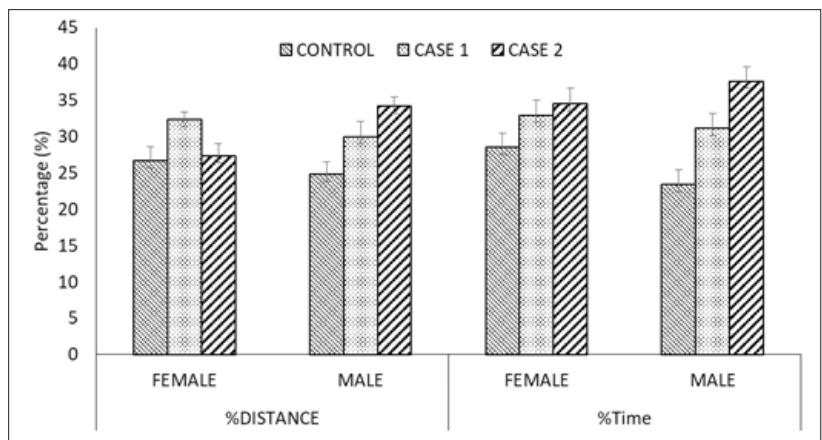

The figure represents a comparison of the percentage of average distance and time spent in the target quadrant on the 5 th day (Probe). The comparison of the groups was done by one-way ANOVA, followed by Tukey's test. The data are average \pm SD. The females of CASE 2 that consumed more WK than the other groups showed a higher percentage, but this difference did not reach to statistical significance

Figure 1. Effects of WK consumption by mothers during pregnancy and lactation on their offspring's memory (Probe trial test) in the MWM test in three groups: Control: (20 g of pellet), CASE 1:(fed with WK, $6 \%$ of food intake during pregnancy and lactation), CASE 2: (fed with WK, 9\% of food intake during pregnancy and lactation) 
On the $5^{\text {th }}$ day (Probe) in which the memory test was performed, there was no significant difference between the control group and the cases (Figure 1). Since animal velocity in finding the platform did not show a significant difference at different days, it indicates that the animals activity is not affected by the intake of the WK.

\section{Histological sections of hippocampal samples}

Histological study of the pyramidal cells in the hippocampal CA1 region of female and male pups in the control, CASE 1, and CASE 2 groups demonstrated an increase in the number of cells in the animals of the CASE 2 group compared to the others $(\mathrm{p}<0.05)$ (Figure 2).

In Figure 3, the photomicrographs of the CA1 region of the hippocampus in female rats are depicted in control, CASE 1, and CASE 2 groups, respectively. The normal morphology of pyramidal neurons, including light cytoplasm and large nucleus with prominent nucleolus, was visible in the cells.

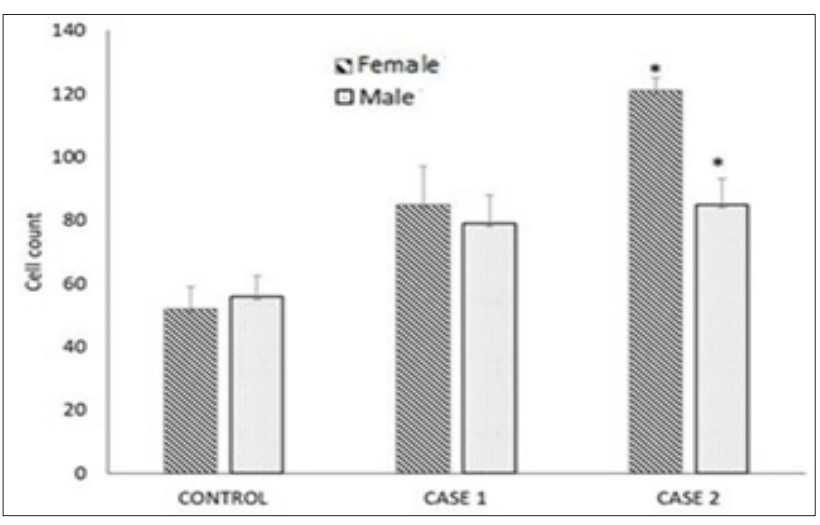

The CASE 2 group that consumed more WK ( $9 \%$ daily intake) than the other groups showed more cell content, which is associated with a significant difference $(p<0.05)$. The comparison of the groups was performed by one-way ANOVA, followed by Tukey 's test. The data are average \pm SD

Figure 2. Effects of WK consumption by mothers during pregnancy and lactation on the number of pyramidal cells in the CA1 region of the hippocampus of their pups (as stained by the H\&E method), in three groups of male and female: as Control: (20 g of pellet), CASE 1: (fed with WK, 6\% of food intake during pregnancy and lactation), CASE 2: (fed with WK, $9 \%$ of food intake during pregnancy and lactation)
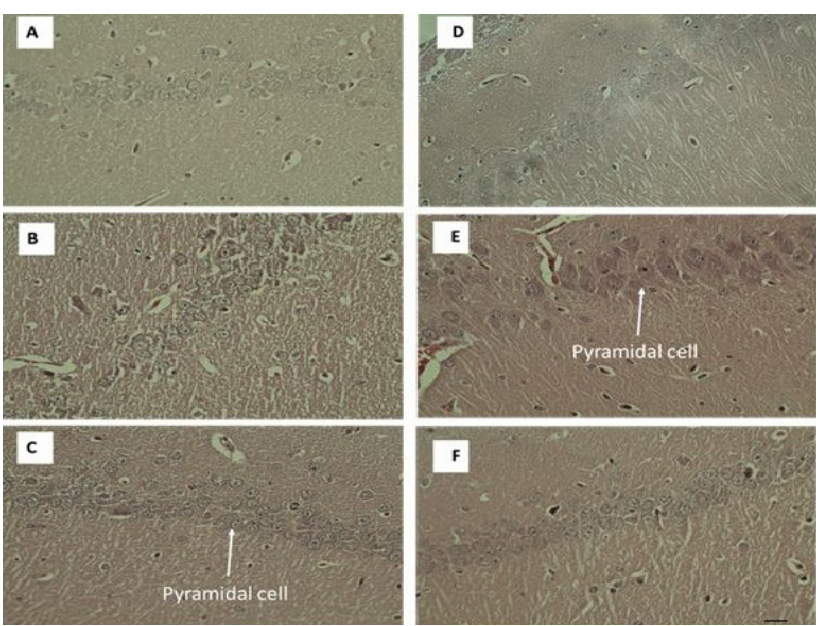

A: Control, B: CASE 1, C: CASE 2 group) and male rats (D: Control, E: CASE 1, F: CASE 2 group), where the pyramidal neurons have a euchoromatic nucleus and large nucleolus stained via the $\mathrm{H} \& \mathrm{E}$ method; Scale bar: $10 \mu \mathrm{m}$

Figure 3. Photomicrograph of the hippocampal CA1 region of female and male offspring

\section{Expression of BDNF and NMDA-R genes in different groups}

BDNF and NMDA-R mRNA expression were evaluated by applying the RT-PCR technique. Here, BDNF and NMDA-R mRNA production were compared with the $\beta$-actin housekeeping gene (Figure 4 and Figure 5). Despite the lack of significant results from statistical analyses in molecular studies, the trend of increase in the expression of BDNF and NMDA-R genes in groups that consumed more WK is evident (Figure 6)

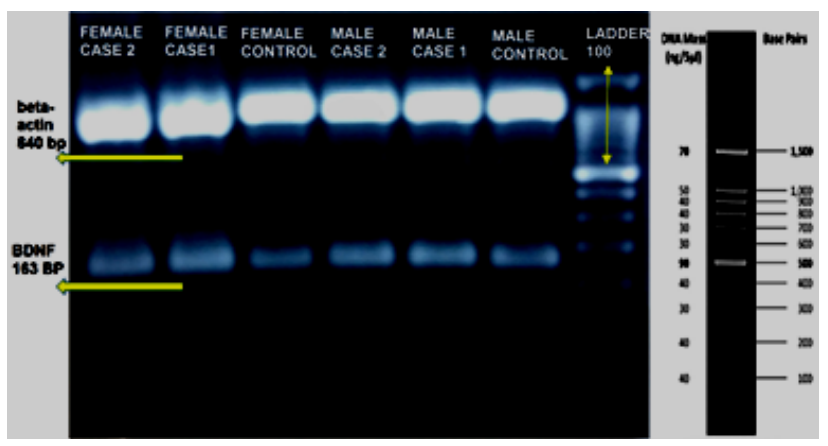

Control: fed with $20 \mathrm{~g}$ of pellet, CASE 1: fed with WK, $6 \%$ of food intake during pregnancy and lactation, CASE 2: fed with WK, $9 \%$ of food intake during pregnancy and lactation). After the extraction of RNA and fabrication of CDNA, PCR reaction and annealing at optimum temperature were performed. Electrophoresis was then done on the gel and the images were analyzed using Image J software

Figure 4. The results of RT-PCR for mRNA of the BDNF gene in male and female rats. RT-PCR results of the effect of WK used by pregnant mothers was related to the expression of BDNF gene in the hippocampus of male and female offspring

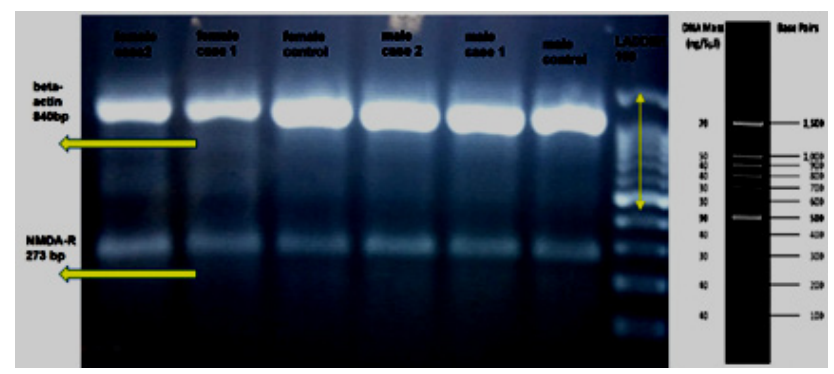

Control: fed with $20 \mathrm{~g}$ of pellet, CASE 1: fed with WK, $6 \%$ of food intake during pregnancy and lactation, CASE 2: fed with WK, $9 \%$ of food intake during pregnancy and lactation) after extraction of RNA and fabrication of CDNA, PCR reaction and annealing at optimum temperature were performed. Electrophoresis was then done on the gel and the images were analyzed using Image J software

Figure 5. The results of RT-PCR for mRNA of the NMDA-R gene in male and female rats. RT-PCR results of the effect of WK used by pregnant mothers was related to the expression of the NMDA-R gene in the hippocampus of male and female offspring

\section{DISCUSSION}

In the current study, the expression of NMDA-R and BDNF genes, learning and memory, and number of neurons of the hippocampal CA1 region were evaluated in adult pups of rats receiving WK or the control diet throughout gestation and lactation. The data showed that animals with higher WK ( $9 \%$ of the daily food) in their diet had female pups with improved spatial learning performance on the MWM. Moreover, the number of pyramidal neurons in the hippocampal CA1 region of male and female pups was increased. 


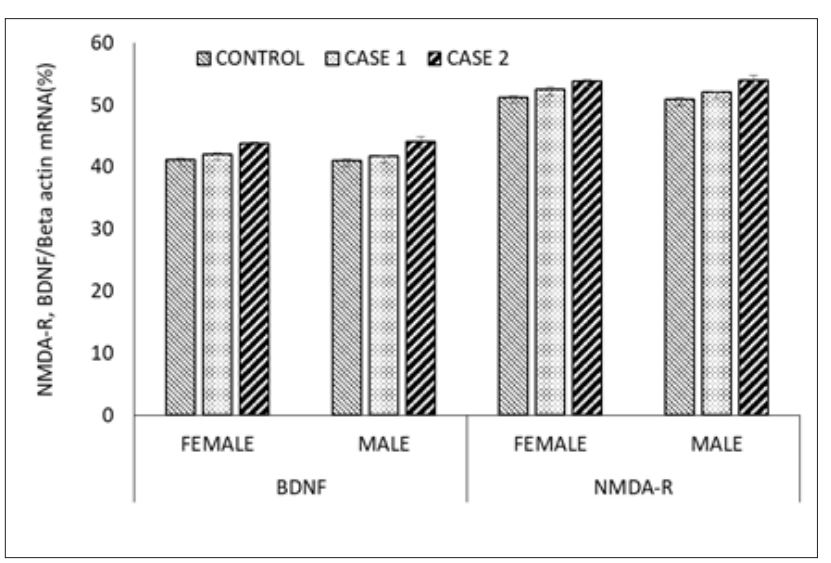

Control: fed with $20 \mathrm{~g}$ of pellet, CASE 1: fed with WK, $6 \%$ of food intake during pregnancy and lactation, CASE 2: fed with WK, $9 \%$ of food intake during pregnancy and lactation). Here, the BDNF and NMDA-R genes were compared with the $\beta$-actin housewife gene. Data is an average \pm standard deviation, expressed as percentages

Figure 6. Gene expression effects of WK used by pregnant mothers was assessed by the expression mRNA of the BDNF and the NMDA-R genes in the hippocampus of male and female offspring

Studies have indicated that WK contains neuroprotective compounds such as vitamin E, folate, melatonin and many polyphenols. It can be stated that this content positively affects learning in rats [9]. Walnut is considered to be a rich source of omega 3 and $\alpha$-Linolenic acid (ALA) [16]. Omega-3 in the central nervous system is responsible for membrane stability, neuroplasticity, signal transduction regulation, serotonin concentration and dopamine regulation [6]. Different studies have shown a positive effect of PUFA on rodent behavior [17]. Furthermore, ALA and LA supplementation have been shown to impact upon learning in rodents, resulting in improved performance of diverse learning tests [18].

Maternal FAs nutrition during pregnancy and lactation determines the transfer of LA and ALA through the placenta and milk [19]. Although lipid transfer through the placenta is very limited, changes in dietary FAs have implications in fetal central nervous system (CNS) development [20]. In fact, the essential FAs provide the precursors for eicosanoids (arachidonic acid, AA) and are important constituents of cell membranes, especially those in the brain tissue. The type and amount of FAs in the diet may modify the brain membrane lipid composition involved in cognitive behavior [21], and a large body of literature states that dietary antioxidants can ameliorate cognitive performance [22].

Our histological evaluation demonstrated that the number of pyramidal neurons in the pups significantly increased in the WK diet ( $9 \%$ of the daily food) group compared to the control. Hence, we may conclude that the use of WK by the mother had influenced upon the neurogenesis of the neurons in the region of the hippocampus (especially in the female), and this finding is of foremost importance and needs more careful consideration.

High amounts of DHA are stored in brain membrane phospholipids during fetal and neonatal development, paralleling membrane expansion in neurogenesis, and synaptogenesis [19]. As mammalian cells do not have the enzymes to form FAs, all of the DHA in the brain must originated from fatty acids in the diet. Before birth, all of the DHA stored in the fetal brain therefore, originates from FAs in the maternal diet via placental transfer, and is either transferred or synthesized in the fetal compartment from ALA derived from the mother. Indeed, various studies have indicated that deprivation of FAs during development results in decreased DHA in brain membrane phospholipids, reduced performance in learning tasks, and altered metabolism of numerous neurotransmitters, including dopamine [23]. Regarding the developing brain, a doubling of membrane phospholipids is required in the $\mathrm{S}$ phase of mitosis for the creation of daughter cells [24].

Synaptic ductility is in fact the same structural and functional changes in the pre-synaptic and post-synaptic components, ranging from short to long term. In most forms of synaptic plasticity, glutamate receptors are involved. Glutamate receptors are activated by adhering glutamate to the protein structure. Unlike glutamate ionotropic receptors, kinetic metabotropic receptors have a very slow activation and effect. As a result, in synaptic base transfer processes, synaptic amplification or weakening, the ionotropic receptors plays a central role, and the metabotropic receptors act as regulators and influencers [25]. In this way, interventions in this process and facilitation of this connection can be applied to the creation of learning and memory.

There are numerous studies on the effects of bio-nutritional compounds on the brain, the results of which have noted highly beneficial effects on both brain and cognitive performance [6,22]. For example, Miller et al. (2014) saw positive effects of WK consumption on the cognitive functions of the brain, such as memory, motor ability, speed, and cognition [26]. Moreover, Asadi et al. (2013) showed that administration of WK to mothers can improve the learning process in their pups [9], although the results of the present study were not fully consistent with their research, and the difference may be due to the type of WK.

In another part of the study, there were possible changes in the pups in the level of expression of the genes involved in memory and learning. The BDNF gene was one of the candidates for molecular study in this research whose expression increased (not significantly) in the case groups. It is involved in the expansion and furcating of dendrites and axons, the maturity of stimulation and inhibition of synapses, and defects causing learning disruption and spatial memory.

The next candidate in the molecular study in the present study was the NMDA-R gene. This gene is an important and abundant nerve mediator receptor of amino acid glutamate in the brain, and acts within the synapses of the pyramidal cells of the CA1 region of the hippocampus to form and integrate synapse. The expression of this gene contributes to the preservation of plasticity and memory. Indeed, in a study by Paoletti et al. (2013), NMDA-R gene disruption caused the destruction of synaptic plasticity and memory impairment in individuals [14]. The results of the PCR molecular test in this study revealed a relative increasing in the gene (not significantly) in the WK consumer groups, probably due to the effect of the bioactive compounds in the WK as transcription factors in the expression of the NMDA-R gene. 


\section{CONCLUSION}

This study has revealed that maternal feeding with WK has useful effects on learning in female pups and in neurogenesis in male and female pups. These effects were more obvious when higher doses of WK were supplemented to mothers. Despite the valuable effects of WK supplementation in the present study, its precise mechanism of action is not known and requires complementary studies for elucidation.

\section{ACKNOWLEDGMENT}

The data presented in this article are based on a master's thesis written by Zahra Mahmoodian in the International campus, Iran University of Medical Sciences, Tehran, Iran.

\section{SOURCE OF FINANCIAL SUPPORT}

This study was supported by a grant from Neuroscience Research Center of Kerman University of Medical Sciences (KUMS).

\section{CONFLICT OF INTEREST}

There is not any conflict of interest in this study.

\section{ORCID iDs}

Zahra Mahmoodian (Dhttps://orcid.org/0000-0002-9270-259X Majid Asadi Shekaari (Dhttps://orcid.org/0000-0003-4367-2280 Mansooreh Soleimani Dhttps://orcid.org/0000-0003-1482-6519 Meysam Ahmadi-Zeidabadi

(Dhttps://orcid.org/0000-0002-4196-2893

Fatemeh Moradi (1)https://orcid.org/0000-0001-7297-5112

Zeinab Akbarnejad (1) https://orcid.org/0000-0002-0822-408X

Khadijeh Esmaeilpour (Dhttps://orcid.org/0000-0002-5349-9285

Taj Pri Kalantaripour (Dhttps://orcid.org/0000-0002-8617-5252

\section{REFERENCES}

1. Toole MJ, Renzaho A. Transient benefits in young children of a nutrition intervention during pregnancy. The Lancet Glob Health. 2014;2(11):e621-e2.

2. Cunha AJLAd, Leite ÁJM, Almeida ISd. The pediatrician's role in the first thousand days of the child: the pursuit of healthy nutrition and development. J Pediatr (Rio).2015;91(6):S44-S51.

3. Akerele OA, Cheema SK. A balance of omega-3 and omega- 6 polyunsaturated fatty acids is important in pregnancy. J Nutr Intermed Metab. 2016;5:23-33.

4. Sherry C, Oliver J, Marriage B. Docosahexaenoic acid supplementation in lactating women increases breast milk and plasma docosahexaenoic acid concentrations and alters infant omega 6: 3 fatty acid ratio. Prostaglandins Leukot Essent Fatty Acids. 2015;95:63-9.

5. Rehman MU, Wali AF, Ahmad A, Shakeel S, Rasool S, Ali R, et al. Neuroprotective strategies for neurological disorders by natural products: An update. Curr Neuropharmacol. 2019;17(3):247-67.

6. Poulose SM, Miller MG, Shukitt-Hale B. Role of Walnuts in maintaining brain health with age. J Nutr. 2014;144(4):561S-6S.

7. Pribis, P, Bailey RN, Russell, AA, Kilsby MA, Hernandez M, Craig WJ, et al. Effects of walnut consumption on cognitive performance in young adults. Br J Nutr. 2012;107(09):1393-401.
8. Asadi-Shekaari, M, Kalantaripour TP, Nejad FA, Namazian E, Eslami A. The anticonvulsant and neuroprotective effects of walnuts on the neurons of rat brain cortex. Avicenna J Med Biotechnol. 2012;4(3):155.

9. Asadi-Shekaari, M, Karimi A, Shabani M, Sheibani V, Esmaelipour K. Maternal feeding with walnuts (Juglans regia) improves learning and memory in their adult pups. Avicenna J Phytomed. 2013;3(4):341-6.

10. Duvernoy, HM. The human brain: surface, three-dimensional sectional anatomy with MRI, and blood supply. Springer Science \& Business Media; 2012.

11. Henke K, Buck A, Weber B, Wieser HG. Human hippocampus establishes associations in memory. Hippocampus. 1997;7(3):249-56.

12. Guo T, Winterburn JL, Pipitone J, Duerden EG, Park MTM, Chau V, et al. Automatic segmentation of the hippocampus for preterm neonates from early-in-life to term-equivalent age. Neuro Image Clin. 2015;9:176-93.

13. Yamada K, Mizuno M, Nabeshima T. Role for brain-derived neurotrophic factor in learning and memory. Life Sci. 2002;70(7): 735-44.

14. Paoletti P, Bellone C, Zhou Q. NMDA receptor subunit diversity: impact on receptor properties, synaptic plasticity and disease. Nat Rev Neurosci. 2013;14(6):383-400.

15. Zihayat B, Khodadadi A, Torabi M, Mehdipour M, Basiri M, Asadi-Shekaar M. Wound healing activity of sheep's bladder extracellular matrix in diabetic rats. Biomed Eng-App Bas C. 2018; 30(2):18500151-8.

16. Haider S, Batool Z, Tabassum S, Perveen T, Saleem S, Naqvi F, et al. Effects of walnuts (Juglans regia) on learning and memory functions. Plant Foods Hum Nutr. 2011;66(4):335-40.

17. Guesnet P, Alessandri J-M. Docosahexaenoic acid (DHA) and the developing central nervous system (CNS)-implications for dietary recommendations. Biochimie. 2011;93(1):7-12.

18. Umezawa M, Ohta A, Tojo H, Yagi H, Hosokawa M, Takeda T. Dietary a-linolenate/linoleate balance influences learning and memory in the senescence-accelerated mouse (SAM). Brain Res. 1995;669(2):225-33.

19. Innis SM. Fatty acids and early human development. Early Hum Dev. 2007;83(12):761-6.

20. Herrera E. Implications of dietary fatty acids during pregnancy on placental, fetal and postnatal development - a review. Placenta. 2002; 23:S9-S19.

21. de Souza AS, Rocha MS, do Carmo MdGT. Effects of a normolipidic diet containing trans fatty acids during perinatal period on the growth, hippocampus fatty acid profile, and memory of young rats according to sex. Nutrition. 2012;28(4):458-64.

22. Kesse-Guyot E, Fezeu L, Jeandel C, Ferry M, Andreeva V, Amieva H, et al. French adults' cognitive performance after daily supplementation with antioxidant vitamins and minerals at nutritional doses: a post hoc analysis of the Supplementation in Vitamins and Mineral Antioxidants (SU. VI. MAX) trial. Am J Clin Nutr. 2011;94(3):892-9.

23. Zimmer L, Vancassel S, Cantagrel S, Breton P, Delamanche S, Guilloteau D, et al. The dopamine mesocorticolimbic pathway is affected by deficiency in $\mathrm{n}-3$ polyunsaturated fatty acids. Am J Clin Nutr. 2002;75(4):662-7.

24. Jackowski S. Cell cycle regulation of membrane phospholipid metabolism. J Biol Chem. 1996;271(34):20219-25.

25. Liu L, Wong TP, Pozza MF, Lingenhoehl K, Wang Y, Sheng M, Auberson YP, Wang YT. Role of NMDA receptor subtypes in governing the direction of hippocampal synaptic plasticity. Science. 2004;304(5673):1021-4.

26. Miller MG, Thangthaeng N, Poulose SM, Shukitt-Hale B. Role of fruits, nuts, and vegetables in maintaining cognitive health. Exp Gerontol. 2017;94:24-28. 